\title{
STABLE AND REGULAR REACHABILITY FOR RELAXED HEREDITARY DIFFERENTIAL SYSTEMS*
}

\author{
FRITZ COLONIUS $\dagger$
}

\begin{abstract}
We investigate reachability properties of relaxed nonlinear hereditary differential systems in the state space $W^{n, \infty}[-r, 0]$. Reachability of the relaxed system is equivalent to approximate reachability of the ordinary system. Local reachability for the relaxed system can be obtained under much weaker conditions than for the ordinary system.

The notion of stable reachability allows us to relate reachability of points in $\mathbb{R}^{n}$ and states in $W^{n, \infty}[-r, 0]$. Regular reachability is of primary importance in optimal control problems with function space end condition, because in case of regularity Lagrange multipliers in the dual of $W^{n, \infty}[-r, 0]$ can be identified with functions in $W^{n, \infty}[-r, 0]$. Regularly reachable final states and regular trajectories are characterized and it is shown that regularity is a generic property of trajectories. Examples are given where all trajectories reaching a certain final state are regular.
\end{abstract}

Introduction. In this paper, we study reachability properties of the following nonlinear hereditary differential system $\Sigma$ :

$$
\begin{array}{ll}
\dot{x}(t)=f\left(x_{t}, u(t), t\right) & \left(t \in T:=\left[t_{0}, t_{1}\right]\right), \\
x_{t_{0}}=\varphi_{0}, & \\
u(t) \in \Omega(t) & (t \in T),
\end{array}
$$

where $0 \leqq r<\infty, t_{1}-r>t_{0}, f: C^{n}[-r, 0] \times \mathbb{R}^{m} \times T \rightarrow \mathbb{R}^{n}, \Omega(t) \subset \mathbb{R}^{m}$ and $\varphi_{0} \in C^{n}[-r, 0]$ are fixed and

$$
x_{t}(s):=x(t+s), \quad s \in[-r, 0] .
$$

$r$ denotes the length of the system memory. The state of this system is given by the function segment $x_{t}$ and the reachability theory depends essentially on the choice of the infinite dimensional state space $Z$.

Jacobs/Kao [15], Banks/Jacobs/Langenhop [2], [3], Colonius/Hinrichsen [11] dealt with complete exact reachability in the Sobolev space $Z=W^{n, 2}[-r, 0]$ for unconstrained linear systems. This work was primarily motivated by fixed final state optimal control problems with unconstrained (resp. energy constrained) controls $u \in L_{2}^{m}(T)$. A natural choice of $Z$ for problems with pointwise constrained controls in $L_{\infty}^{m}$-as considered here-is $Z=W^{n, \infty}[-r, 0]$. (This compatibility requirement on the state and control function spaces is exposed very clearly by Kurcyusz/Olbrot [17].)

However, as is well known, there is a severe drawback of reachability theory in $W^{n, p}[-r, 0], 1 \leqq p \leqq \infty$ : complete exact reachability for linear systems can only be guaranteed if the dimension $m$ of the control space is not less than the dimension $n$ of the phase space. Hence it appears reasonable to replace exact reachability by approximate reachability. This concept, in fact, has been studied for unconstrained linear systems by many authors (see, e.g., Delfour/Mitter [12], Manitius/Triggiani [20], Manitius [19] in $Z=\mathbb{R}^{n} \times L_{2}^{n}[-r, 0]$, Olbrot [23] in various Banach spaces). While this turned out successful for certain purposes (e.g., feedback stabilizability and the infinite time linear quadratic optimal control problem), it is inadequate for fixed final state optimal control problems. For these problems exact reachability of the linearized system is necessary in order to guarantee the existence of Lagrange multipliers (see

\footnotetext{
* Received by the editors November 11, 1980, and in final revised form October 17, 1981.

$\dagger$ Forschungsschwerpunkt Dynamische Systeme, Universität Bremen, Bibliothekstraße, Postfach 330 440, 2800 Bremen 33, West Germany.
} 
Kurcyusz [16]). Furthermore, with respect to the phase space $Z=W^{n, \infty}[-r, 0]$, Olbrot [23, Thm. 5.3] has shown that for linear unconstrained systems approximate reachability is equivalent to exact reachability; i.e., nothing is gained by allowing approximate reachability. In this paper we propose the following way out of this apparent dilemma: we study instead of the system $(0.1),(0.2),(\widetilde{0.3})$ the relaxed system in the sense of Warga [27]. That is, we consider instead of measurable control functions $u$ satisfying (0.3) relaxed (measure-valued) control functions

$$
v \in \mathscr{S}^{\#}
$$

and replace $u$ in $(0.1)$ by $v$.

Exact reachability with relaxed controls is equivalent to approximate reachability with ordinary pointwise constrained controls (see Theorem 2.1 and Remark 2.1 below).

It is well known that for linear systems final states which are not exactly reachable can only be approximated by applying an unbounded sequence of control functions (see Kurcyusz [16]). We show, however, that neither this nor Olbrot's result on the equivalence of exact and approximate reachability in $W^{n, \infty}[-r, 0]$ remains true if the control appears nonlinearly. Here local reachability of the relaxed system is a much weaker property than local reachability of the ordinary system. In particular, the condition $m \geqq n$ on the dimensions $m$ and $n$ of the control and phase space, respectively, is not necessary for local reachability of the relaxed system. The consequences for optimal control problems are studied in the companion paper [10].

Since the controls are bounded we study local reachability. Now suppose we can reach a certain final state $\varphi_{1}$ with a trajectory $x^{0}$ corresponding to a control $v^{0} \in \mathscr{S}^{\#}$. Then in order to reach $\varphi$ in a neighborhood of $\varphi_{1}$ in $W^{n, \infty}[-r, 0]$, we first have to reach $\varphi(-r)$ at time $t_{1}-r$ and then trace exactly the velocity function $\dot{\varphi}\left(t-t_{1}\right)$ on $\left[t_{1}-r, t_{1}\right]$.

In order to achieve this we introduce the concept of stable reachability. This means that a complete neighborhood of $\varphi_{1}(-r) \in \mathbb{R}^{n}$ can be reached with arbitrarily small deviations from $x^{0}$. Then the hereditary effects influencing the velocity on $\left[t_{1}-r, t_{1}\right]$ can be kept small, and in order to reach a complete neighborhood of $\varphi_{1}$ in $W^{n, \infty}[-r, 0]$ we only have to compensate small deviations from $\varphi_{1}=x_{t_{1}}^{0}$.

If a complete neighborhood of $\dot{\varphi}_{1}\left(t-t_{1}\right)=\dot{x}^{0}(t)=f\left(x_{t}^{0}, v^{0}(t), t\right), t \in\left[t_{1}-r, t_{1}\right]$ in $L_{\infty}^{n}\left[t_{1}-r, t_{1}\right]$ can be covered by altering the control $v^{0}$ only on $\left[t_{1}-r, t_{1}\right]$, we say that $\varphi_{1}$ is reached regularly with $x^{0}$ (for exact definitions see Definitions $2.1,2.2,3.1$ ). The notion of regular reachability is of primary importance for optimal control problems with function space end condition, because in case of regularity Lagrange multipliers in the dual of $W^{n, \infty}[-r, 0]$ can be identified with functions in $W^{n, \infty}[-r, 0]$ (see Colonius [9], [10] (this issue, pp. 695-712)). The analysis of the somewhat delicate relations among local, stable and regular reachability constitutes the main content of this paper. The results extend and sharpen those of Colonius [9]. After the preliminary $\S 1$, we discuss in $\S 2$ the relation between stable and local reachability. In particular, it turns out that both notions are equivalent for linear relaxed systems, while for nonlinear systems there may be final states which are locally but not stably reachable. In $\S 3$, we show that regular reachability plus a stable reachability property in $\mathbb{R}^{n}$ implies stable reachability in $W^{n, \infty}[-r, 0]$. This, conversely, does not imply regular reachability, but a weaker property. For linear systems of the form (see (3.4) below)

$$
\dot{x}(t)=L(t) x_{t}+B(t) u(t)
$$

this property specializes to the well-known rank condition for $B(t)$ on $\left[t_{1}-r, t_{1}\right]$ plus essential boundedness of the generalized inverse $B(t)^{+}$on $\left[t_{1}-r, t_{1}\right]$. This proves, in 
the state space $W^{n, \infty}[-r, 0]$, a conjecture by Banks/Jacobs/Langenhop [3]. In $\S 4$, attention is focused on properties of the whole reachable set $\mathscr{R}$ and of the set $\mathscr{T}$ of trajectories. The subsets of regularly reachable states in $\mathscr{R}$ and of regular trajectories in $\mathscr{T}$ are described. It is shown that regularity is a generic property of trajectories. Examples are given where all trajectories reaching a certain final state are regular.

Notation and conventions. The Banach space of continuous functions on the compact set $A \subset \mathbb{R}^{m}$ with values in $\mathbb{R}^{n}$ is denoted by $C^{n}(A)$. For $1 \leqq p \leqq \infty, W^{n, p}[a, b]$ denotes the Sobolev space of absolutely continuous functions $x:[a, b] \rightarrow \mathbb{R}^{n}$ with derivative $\dot{x} \in L_{p}^{n}[a, b]$, that is, with $p$-integrable, respectively essentially bounded derivative. The norm in the Banach space $W^{n, p}[a, b]$ is given by $\|x\|:=\left|\left(|x(a)|,\|\dot{x}\|_{L_{p}}\right)\right|$, where $|\cdot|$ denotes the Euclidean norm in finite dimensional space. $W^{n, p}[a, b]$ is identified in the canonical way with $\mathbb{R}^{n} \times L_{p}^{n}[a, b]$. The topological dual of a Banach space $X$ is denoted by $X^{*}$. The interior of a set $A$ in a Banach space is denoted by int $A$ and $\operatorname{co} A$ is its convex hull. For $\delta>0$, int ${ }_{\delta} A$ is the set of all points in int $A$ having at least distance $\delta$ to the boundary $\partial A$ of $A$.

$\mathscr{L}\left(X_{1}, X_{2}\right)$ is the space of bounded linear operators from a Banach space $X_{1}$ into a Banach space $X_{2}$. For a measurable subset $S \subset \mathbb{R}$, we write $(s \in S)$ instead of for Lebesgues almost all $s \in S$. For the sets $\Omega(t)$ of admissible control values we assume that $\Omega(t) \subset \Omega_{0}(t \in T)$, where $\Omega_{0} \subset \mathbb{R}^{m}$ is compact, $t \mapsto \Omega(t)$ is measurable, and $\Omega(t)$ is closed for almost all $t \in T$. The set of Radon probability measures on $\Omega_{0}$ is denoted by $\operatorname{rpm}\left(\Omega_{0}\right)$. The set of relaxed controls $v$ defined on the fixed time interval $T:=\left[t_{0}, t_{1}\right]$ with values in the set of Radon probability measures on $\Omega_{0}$ having support contained in $\Omega(t)$ is denoted by $\mathscr{S}^{\#}$. Then $\mathscr{S}^{\#}$ can be identified with a subset of the dual space $\mathcal{N}:=L_{1}\left(T, C\left(\Omega_{0}\right)\right)^{*}$ and is considered in the weak* topology (for all this see Warga [27]).

Relaxed controls satisfy the following weak measurability requirement:

$$
t \mapsto c(v(t)):=\int_{\Omega_{0}} c(\omega) v(t)(d \omega)
$$

is measurable for each $c \in C\left(\Omega_{0}\right)$.

For $f$ as in Lemma 1.1 below we define

$$
f\left(x_{t}, v(t), t\right):=\int_{\Omega_{0}} f\left(x_{t}, \omega, t\right) v(t)(d \omega),
$$

and identify an ordinary control $u$ with the relaxed control $\delta_{u(\cdot)} \in \mathscr{S}^{\#}$, where $\delta_{u(t)}$ denotes the point measure concentrated at $u(t) \in \Omega(t) . T_{1}$ denotes the final interval $\left[t_{1}-r, t_{1}\right]$. Since the initial state $\varphi_{0}$ of the relaxed system $\Sigma$ described by $(0.1),(0.3)$ remains fixed, we tacitly assume that $x$ satisfies $x_{t_{0}}=\varphi_{0}$, when we speak of "a trajectory $x$ of $\Sigma$ '. A pair $(x, v)$ is called a solution of $\Sigma$, if $v \in \mathscr{S}^{\#}$ and $x$ satisfies $(0.1),(0.2)$.

1. Preliminaries. In this section we formulate conditions on $f$ which will be needed in the sequel, and we investigate how the right-hand side of $(0.1)$ depends on trajectories and controls. Then it is shown that the relaxation of the problem is equivalent to the convexification of the set of velocity vectors. Proofs are very condensed or omitted (see Colonius [8]).

LEMMA 1.1. Assume that the following conditions on $f$ are satisfied:

(1.1) The function $f: C^{n}[-r, 0] \times \mathbb{R}^{m} \times T \rightarrow \mathbb{R}^{n}$ is continuous in $(\varphi, \omega) \in$ $C^{n}[-r, 0] \times \mathbb{R}^{m}$ and measurable in $t \in T$;

(1.2) There is $p: \mathbb{R}_{+} \times T \rightarrow \mathbb{R}_{+}$such that for all $x \in C^{n}\left[t_{0}-r, t_{1}\right]$ and $\omega \in \Omega_{0}$

$$
\left|f\left(x_{t}, \omega, t\right)\right| \leqq p\left(\left\|x_{t}\right\|_{\infty}, t\right) \quad(t \in T),
$$


where $p(s, \cdot) \in L_{\infty}^{1}(T)$ for all $s \in \mathbb{R}_{+}$and $p(\cdot, t)$ is monotonically increasing for almost all $t \in T$.

Let $\left(\left(x^{k}, v^{k}\right)\right) \subset C^{n}\left[t_{0}-r, t_{1}\right] \times \mathscr{S}^{\#}$ be a sequence with $x^{k} \rightarrow x^{0}$ in $C^{n}\left[t_{0}-r, t_{1}\right]$ and $v^{k} \rightarrow v^{0}$ weakly* in $\mathscr{S}^{\#}$. Then $\left(f\left(x_{t}^{k}, v^{k}(t), t\right), t \in T\right) \rightarrow\left(f\left(x_{t}^{0}, v^{0}(t), t\right), t \in T\right)$ weakly* in $L_{\infty}^{n}(T)$, hence weakly in $L_{2}^{n}(T)$.

If $p(s, \cdot)$ in $(1.2)$ is only known to be a $L_{2}$-function, the convergence property still holds in the weak $L_{2}$-topology.

Variants of this lemma implying weak $L_{2}$-convergence are well known, even for functions $f$ allowing lags in the controls (Berkovitz [5], Warga [26], Bates [4]). Weak* convergence in $L_{\infty}$ follows similarly.

LEMMA 1.2. Assume that (1.1) and (1.2) hold and moreover the following condition is satisfied:

(1.3) The function $f$ is continuously Fréchet differentiable in the first argument, the corresponding derivative $D_{1} f(\varphi, \omega, t)$ is continuous in $(\varphi, \omega, t)$ and for all $\omega \in \Omega_{0}$

where $p$ is as in (1.2).

$$
\left\|D_{1} f(\varphi, \omega, t)\right\| \leqq p\left(\|\varphi\|_{\infty}, t\right) \quad(t \in T)
$$

Let $\left(\left(x^{k}, v^{k}\right)\right) \subset C^{n}\left[t_{0}-r, t_{1}\right] \times \mathscr{S}^{\#}$ be a sequence with $x^{k} \rightarrow x^{0}$ in $C^{n}\left[t_{0}-r, t_{1}\right]$ and $v^{k} \rightarrow v^{0}$ in the strong norm topology on $\mathscr{S}^{\#}$ (see Warga [27, Thm. IV.1.9]. Then

$$
\text { ess sup }\left|f\left(x_{t}^{k}, v^{k}(t), t\right)-f\left(x_{t}^{0}, v^{0}(t), t\right)\right| \rightarrow 0
$$

and the Fréchet derivatives $D_{1} f\left(\varphi, v^{k}(t), t\right)$ exist and have the form $\left(\psi \in C^{n}[-r, 0]\right)$

$$
D_{1} f\left(\varphi, v^{k}(t), t\right) \psi=\int_{\Omega_{0}} D_{1} f(\varphi, \omega, t) v^{k}(t)(d \omega)
$$

furthermore

$$
\text { ess sup }\left\|D_{1} f\left(x_{t}^{k}, v^{k}(t), t\right)-D_{1} f\left(x_{t}^{0}, v^{0}(t), t\right)\right\| \rightarrow 0 .
$$

Proof. Using (1.1)-(1.3) and the mean value theorem, we find

$$
\begin{aligned}
& \left\|f\left(x_{t}^{k}, v^{k}(t), t\right)-f\left(x_{t}^{0}, v^{0}(t), t\right)\right\|_{\infty} \\
& \quad \leqq \underset{t, \omega}{\operatorname{ess} \sup }\left|f\left(x_{t}^{k}, \omega, t\right)\left\|v^{k}-v^{0}\right\|+\underset{t, \omega}{\operatorname{ess} \sup }\right| f\left(x_{t}^{k}, \omega, t\right)-f\left(x_{t}^{0}, \omega, t\right) \mid \\
& \quad \leqq \operatorname{ess} \sup _{t} p\left(c_{0}, t\right)\left\|v^{k}-v^{0}\right\|+\operatorname{ess} \sup _{t} p\left(c_{0}, t\right)\left\|x^{k}-x^{0}\right\|_{\infty}
\end{aligned}
$$

for a constant $c_{0}>0$. The right-hand side converges to 0 by assumption. The existence and the form of the derivatives $D_{1} f\left(\varphi, v^{k}(t), t\right)$ follow by Warga [27, Thm. II.3.10]. For $\psi \in C^{n}[-r, 0]$ with $\|\psi\|_{\infty} \leqq 1$

$$
\begin{aligned}
& \left\|D_{1} f\left(x_{t}^{k}, v^{k}(t), t\right) \psi-D_{1} f\left(x_{t}^{0}, v^{0}(t), t\right) \psi\right\| \\
& \quad \leqq \sup _{t, \omega}\left\|D_{1} f\left(x_{t}^{k}, \omega, t\right)-D_{1} f\left(x_{t}^{0}, \omega, t\right)\right\|\left\|v^{k}\right\|+\sup _{t, \omega}\left\|D_{1} f\left(x_{t}^{0}, \omega, t\right)\right\|\left\|v^{k}-v^{0}\right\| .
\end{aligned}
$$

This converges to 0 , since $\Omega \times T$ is compact and $D_{1} f$ is continuous.

LEMMA 1.3. Let the conditions (1.1) and (1.2) be satisfied and assume that the trajectories of $\Sigma$ are uniformly bounded. Then the set $\mathscr{T}$ of trajectories $x$ of $\Sigma$ is compact and sequentially compact in the weak ${ }^{*}$ topology of $W^{n, \infty}(T)$ and the uniform norm topology.

Proof. Sequential weak* compactness of $\mathscr{T}$ follows by sequential weak* compactness of $\mathscr{S}^{\#}$, Warga [27, Thm. IV.3.11, and Lemma 1.1]. Since $W^{n, \infty}$ is the dual of the separable space $W^{n, 1}$, weak* compactness of $\mathscr{T}$ is equivalent to weak* sequential 
compactness (see Dunford/Schwartz [13, p. 437]. Finally, compactness in the topology of uniform convergence follows, because the embedding of $W^{n, \infty}(T)$ into $C^{n}(T)$ is compact.

Remark 1.1. The trajectories of $\Sigma$ are uniformly bounded, if (1.3) is satisfied. The following lemma allows us to characterize relaxed velocity vectors.

LEMMA 1.4. For a measurable subset $S \subset T$, consider a measurable function $z: S \rightarrow$ $\mathbb{R}^{n}$ and a function $\Phi: \Omega_{0} \times S \rightarrow \mathbb{R}^{n}$ with $\Phi(\omega, \cdot)$ measurable for all $\omega \in \Omega_{0}$ and $\Phi(\cdot, t)$ continuous for a.a. $t \in S$. Then the following three conditions are equivalent:

$$
\begin{array}{ll}
z(t) \in \operatorname{co} \Phi(\Omega(t), t) & (t \in S) ; \\
z(t)=\Phi(v(t), t) & (t \in S)
\end{array}
$$

for an element $v \in \mathscr{S}^{\#}$;

$$
z(t)=\sum_{i=0}^{n} \alpha_{i}(t) \Phi\left(u_{i}(t), t\right) \quad(t \in S)
$$

for some measurable $\alpha_{i}: T \rightarrow \mathbb{R}_{+}$and $u_{i}: T \rightarrow \Omega_{0}$ with $\sum_{i=0}^{n} \alpha_{i}(t)=1$ and $u_{i}(t) \in \Omega(t)$.

Proof. The proof follows by Warga [27, Thms. I.6.13, IV 3.13; compare also Thm. VI.3.2].

Remark 1.2. Consider

$$
\left\{\left(f\left(x_{t}, v(t), t\right), t \in T\right): v \in \mathscr{S}^{\#}\right\} .
$$

Then the lemma above implies that this set coincides with

$$
\left\{z \in L_{\infty}^{n}(T): z(t) \in \operatorname{co} f\left(x_{t}, \Omega(t), t\right)(t \in T)\right\} .
$$

This shows that along a fixed trajectory $x$ the set of relaxed velocity vectors coincides with the convex hull of the set of ordinary velocity vectors. Hence the relaxed system $\Sigma$ is equivalent to the relaxed system considered by Oguztöreli [22, § 8.9].

LEMMA 1.5. Assume that (1.1)-(1.3) hold and, additionally, that the following condition is satisfied:

(1.4) For each relaxed control $v \in \mathscr{S}^{\#}$ there is a unique trajectory $x=S(v) \in$ $C^{n}\left[t_{0}-r, t_{1}\right]$ satisfying $(0.1)$ and $(0.2)$.

Then $\boldsymbol{S}(v)$ depends in a continuously Fréchet differentiable manner on $v \in \mathscr{S}^{\#}$; for $v, v^{0} \in \mathscr{S}^{\#}$ and $x^{0}:=S\left(v^{0}\right)$, the trajectory $x(v):=D S\left(v^{0}\right)\left(v-v^{0}\right) \in C^{n}\left[t_{0}-r, t_{1}\right]$ satisfies

$$
\begin{aligned}
& \dot{x}(t)=D_{1} f\left(x_{t}^{0}, v^{0}(t), t\right) x_{t}+f\left(x_{t}^{0}, v(t)-v^{0}(t), t\right) \quad(t \in T), \\
& x_{t_{0}}=0 .
\end{aligned}
$$

Proof. The proof follows by the implicit function theorem and computation of derivatives according to Lemma 1.2.

Remark 1.3. It is convenient to write the linearized system in the form (1.5), because (1.5) involves the relaxed control $v \in \mathscr{S}^{\#} ; v-v^{0}$ is not in the set $\mathscr{S}^{\#}$ of relaxed controls.

Remark 1.4. Assumption (1.4) requires existence and uniqueness for trajectories corresponding to relaxed controls. This problem can be reduced to existence and uniqueness theory of functional differential equations using the representation of relaxed velocities introduced by Gamkrelidze: by Lemma 1.4 for each relaxed trajectory $x$ there exist measurable functions $\alpha_{0}, \alpha_{1}, \cdots, \alpha_{n}: T \rightarrow \mathbb{R}_{+}$with $\sum \alpha_{i}(t)=1$ and ordinary controls $u_{0}, u_{1}, \cdots, u_{n}$ with values in $\Omega(t)$ such that

$$
\dot{x}(t)=\sum_{i=0}^{n} \alpha_{i}(t) f\left(x_{t}, u_{i}(t), t\right) \quad(t \in T),
$$


and conversely. Hence relaxed trajectories satisfy a functional differential equation; for results on the solution of these equations see Hale [14].

Remark 1.5. The set of trajectories of (1.5) coincides with the set of trajectories of the system

$$
\dot{x}(t)=D_{1} f\left(x_{t}^{0}, v^{0}(t), t\right) x_{t}+u(t) \quad(t \in T)
$$

where $u: T \rightarrow \mathbb{R}^{n}$ are measurable functions with values $u(t)$ in

$$
\operatorname{co} f\left(x_{t}^{0}, \Omega(t), t\right)-f\left(x_{t}^{0}, v^{0}(t), t\right)=\operatorname{co} f\left(x_{t}^{0}, \Omega(t), t\right)-\dot{x}^{0}(t) .
$$

This is a consequence of Lemma 1.4. Observe that (1.5) (and (1.6)) again is uniquely solvable by Hale [14, Chap. 6, Thm. 2.1].

2. Local and stable reachability. In this section we formally state the relation between the ordinary and the relaxed problem indicated in the introduction. Then we prove sufficiency of a criterion for stable reachability and show that local and stable reachability are equivalent for linear relaxed systems.

In the rest of this paper we assume that the conditions (1.1), (1.2) and (1.4) are satisfied.

THEOREM 2.1. If the trajectories of $\Sigma$ are uniformly bounded, the following assertions hold:

(i) Suppose $\left(x^{0}, v^{0}\right)$ is a solution of $\Sigma$. Then there is a sequence $\left(\left(x^{k}, v^{k}\right)\right)$ of solutions of $\Sigma$, where $v^{k}$ is an ordinary control, such that $v^{k} \rightarrow v^{0}$ weakly ${ }^{*}$ in $\mathscr{S}^{\#}$ and $x^{k} \rightarrow x^{0}$ in $C^{n}\left[t_{0}-r, t_{1}\right]$.

(ii) If, conversely, $\left(\left(x^{k}, v^{k}\right)\right)$ is a sequence of solutions of $\Sigma$ (where $v^{k}$ is not necessarily an ordinary control) with $x_{t_{1}}^{k} \rightarrow \varphi_{1}$ in $C^{n}[-r, 0]$, then there exists a solution $\left(x^{0}, v^{0}\right)$ of $\Sigma$ such that for a subsequence $v^{k} \rightarrow v^{0}$ weakly* in $\mathscr{S}^{\#}$ and $x^{k} \rightarrow x^{0}$ in $C^{n}\left[t_{0}-r, t_{1}\right]$; in particular $x_{t_{1}}^{0}=\varphi_{1}$.

Proof. (i) Since the ordinary controls are dense in $\mathscr{S}^{\#}$ (Warga [27, Thm. IV.3.10]) there is a sequence $\left(v^{k}\right)$ of ordinary controls converging to $v^{0}$. Then a subsequence of the corresponding sequence of trajectories $x^{k}:=S\left(v^{k}\right)$ converges by Lemma 1.3, and by Lemma 1.2 and assumption (1.4) its limit is $x^{0}$.

(ii) follows similarly.

Remark 2.1. The assertions of the theorem remain true if uniform convergence of the trajectories (resp. final states) is replaced by weak* convergence in $W^{n, \infty}$.

The following definition presents an abus de langage; however, it is quite convenient.

Definition 2.1. A function $\varphi_{1} \in W^{n, \infty}[-r, 0]$ is called locally reachable at time $t_{1}$, if and only if there is a neighborhood $N$ of $\varphi_{1}$ in $W^{n, \infty}[-r, 0]$ such that for each $\varphi \in N$ there is a trajectory $x$ of $\Sigma$ with $\varphi=x_{t_{1}}$. Analogously, we define local reachability of a point $\alpha$ in $\mathbb{R}^{n}$.

Definition 2.2. A function $\varphi_{1} \in W^{n, \infty}[-r, 0]$ is called stably reachable at time $t_{1}$ with a trajectory $x^{0}$ of $\Sigma$, if and only if for each neighborhood $V$ of $x^{0}$ in $C^{n}\left[t_{0}-r, t_{1}\right]$ there is a neighborhood $N$ of $\varphi_{1}$ in $W^{n, \infty}[-r, 0]$ such that for each $\varphi \in N$ there is a trajectory $x \in V$ of $\Sigma$ with $\varphi=x_{t}$. Analogously, we define stable reachability of a point $\alpha$ in $\mathbb{R}^{n}$.

Stable reachability of $\varphi$ (resp., $\alpha$ ) with $x^{0}$ means that one can reach a complete neighborhood of $\varphi_{1}$ (resp., $\alpha$ ) with arbitrarily small deviations from $x^{0}$. Obviously this implies local reachability. However, the following example which is taken from Lee/Markus [18, p. 257] illustrates that in general the converse does not hold. It is a nonlinear system with $r=0$, i.e., an ordinary differential system. 
Example 2.1. Consider

$$
\begin{aligned}
& \dot{x}^{1}(t)=x^{2}(t) u^{1}(t)-x^{1}(t) u^{2}(t), \\
& \dot{x}^{2}(t)=-x^{1}(t) u^{1}(t)-x^{2}(t) u^{2}(t), \quad t \in[0, \pi], \\
& x^{1}(0)=1, \quad x^{2}(0)=0, \\
& \left(u^{1}(t), u^{2}(t)\right) \in \Omega:=\left\{\left(\omega_{1}, \omega_{2}\right) \in \mathbb{R}^{2}:\left|\omega_{1}\right| \leqq 1,\left|\omega_{2}\right| \leqq 1\right\} .
\end{aligned}
$$

Since for fixed $x=\left(x^{1}, x^{2}\right)$ the velocity sets are convex, it suffices to consider ordinary controls (see Remark 1.2). In polar coordinates $(r, \varphi)$, the system is described by

$$
\begin{aligned}
& \dot{r}(t)=-r(t) u^{2}(t), \quad \dot{\varphi}(t)=-u^{1}(t), \\
& r(0)=1, \quad \varphi(0)=0 .
\end{aligned}
$$

The reachable set at time $t_{1}=\pi$ is an annulus around 0 with inner radius $e^{-\pi}$ and outer radius $e^{\pi}$. It is not difficult to see that all pairs $(r, \pi)$ with $e^{-\pi}<r<e^{\pi}$ are locally reachable, but not stably reachable at time $t_{1}=\pi$.

Remark 2.2. It seems that this is a general phenomenon: We conjecture that any point being for the first time in the interior of the reachable set without havingbefore that time-been at the boundary of the reachable set is not stably reachable.

Condition (2.2), below, is related to regular reachability (see $\S 3$ ) and will play an important role in the rest of this paper. The following stability lemma shows that it remains valid under small perturbations of the trajectory $x^{0}$.

LEMMA 2.1. Assume that (1.3) and the following condition are satisfied:

(2.2) For a measurable subset $S \subset T$, for $x^{0} \in C^{n}\left[t_{0}-r, t_{1}\right]$, and $z \in L_{\infty}^{n}(S)$, there is a neighborhood $V$ of $0 \in \mathbb{R}^{n}$ such that

$$
V \subset z(t)+\operatorname{co} f\left(x_{t}^{0}, \Omega(t), t\right) \quad(t \in S) .
$$

Then there are $\delta>0$ and a neighborhood $\tilde{V}$ of $0 \in \mathbb{R}^{n}$ such that for all $x$ with $\left\|x-x^{0}\right\|_{\infty}<\delta$

$$
\tilde{V} \subset z(t)+\operatorname{co} f\left(x_{t}, \Omega(t), t\right) \quad(t \in S) .
$$

Proof. Take $V$ as an $n$-simplex with vertices $e_{0}, e_{1}, \cdots, e_{n}$. Then by Lemma 1.4 there are $v_{i} \in \mathscr{S}^{\#}$ with

$$
e_{i}=z(t)+f\left(x_{t}^{0}, v_{i}(t), t\right) \quad(t \in S) .
$$

By Lemma 1.2, $f\left(x_{t}, v_{i}(t), t\right)$ is essentially uniformly close to $f\left(x_{t}^{0}, v_{i}(t), t\right)$ for small $\left\|x-x^{0}\right\|_{\infty}$. Thus there is $\delta>0$ such that for all $x$ with $\left\|x-x^{0}\right\|_{\infty}<\delta$ and for a.a. $t \in S$ the points $f\left(x_{t}, v_{i}(t), t\right), i=0,1, \cdots, n$, are also vertices of $n$-simplices containing a fixed neighborhood $\tilde{V}$ of $0 \in \mathbb{R}^{n}$. This proves the lemma, since convex combinations of $f\left(x_{t}, v_{i}(t), t\right), i=0,1, \cdots, n$ remain in $\operatorname{co} f\left(x_{t}, \Omega(t), t\right)$.

Remark 2.3. Assumption (1.3) may be replaced by continuity of $f(\varphi, \omega, t)$ in $(\varphi, \omega, t)$ (compare the second part of Lemma 1.2).

THEOREM 2.2. Suppose the trajectory $x^{0}$ of $\Sigma$ reaches $\varphi_{1} \in W^{n, \infty}[-r, 0]$ at time $t_{1}$, and there are $\varepsilon>0$ and a neighborhood $V$ of $0 \in \mathbb{R}^{n}$ such that

$$
V \subset-\dot{x}^{0}(t)+\operatorname{co} f\left(x_{t}^{0}, \Omega(t), t\right) \quad\left(t \in\left[t_{1}-r-\varepsilon, t_{1}-r\right]\right) .
$$

Then $\varphi_{1}(-r)$ is stably reachable with $x^{0}$ at time $t_{1}-r$.

If the inclusion in (2.3) holds also for a.a. $t \in T_{1}$, the function $\varphi_{1}$ is stably reachable with $x^{0}$ at time $t_{1}$. 
Proof. By the stability lemma, Lemma 2.1, there are $\delta_{0}>0$ and a neighborhood $U$ of $\dot{x}^{0} \mid\left[t_{1}-r-\varepsilon, t_{1}-r\right] \in L_{\infty}^{n}$ such that for all $x$ with $\left\|x-x^{0}\right\|_{\infty}<\delta_{0}$ and all $z \in U$

$$
z(t) \in \operatorname{co} f\left(x_{t}, \Omega(t), t\right) \quad\left(t \in\left[t_{1}-r-\varepsilon, t_{1}-r\right]\right) .
$$

For all $\delta>0$, the set $N_{\delta}$ defined by

$$
\begin{array}{r}
N_{\delta}:=\left\{\alpha \in \mathbb{R}^{n}: \alpha=x\left(t_{1}-r\right) \text { for some } x \in C^{n}\left[t_{0}-r, t_{1}\right]\right. \\
\text { with } x(t)=x^{0}(t) \text { for } t \in\left[t_{0}-r, t_{1}-r-\varepsilon\right], \\
\left.\left\|x-x^{0}\right\|_{\infty}<\delta \text { and } \dot{x} \mid\left[t_{1}-r-\varepsilon, t_{1}-r\right] \in U\right\}
\end{array}
$$

forms a neighborhood of $\varphi_{1}(-r)$ in $\mathbb{R}^{n}$. For $0<\delta<\delta_{0}$ take $\alpha \in N_{\delta}$. Then there is $x \in C^{n}\left[t_{0}-r, t_{1}\right]$ as in the definition of $N_{\delta}$ with

$$
\dot{x}(t) \in \operatorname{co} f\left(x_{t}, \Omega(t), t\right) \quad\left(t \in\left[t_{1}-r-\varepsilon, t_{1}-r\right]\right) .
$$

By Lemma 1.4, $x$ is a trajectory of $\Sigma$, reaching $\alpha$ by definition. Thus $x^{0}$ reaches $\varphi_{1}(-r)$ stably at time $t_{1}-r$.

Stable reachability of $\varphi_{1}$ follows similarly, since for each $\delta>0$ and each neighborhood $U$ of $\dot{x}^{0} \mid\left[t_{1}-r-\varepsilon, t_{1}\right]$ in $L_{\infty}^{n}$ the set

$$
\begin{aligned}
& \left\{\varphi \in W^{n, \infty}[-r, 0]: \varphi=x_{t_{1}} \text { for some } x \in C^{n}\left[t_{0}-r, t_{1}\right]\right. \\
& \text { with } x(t)=x^{0}(t) \text { for } t \in\left[t_{0}-r, t_{1}-r-\varepsilon\right], \\
& \left.\left\|x-x^{0}\right\|_{\infty}<\delta \text {, and } \dot{x} \mid\left[t_{1}-r-\varepsilon, t_{1}\right] \in U\right\}
\end{aligned}
$$

forms a neighborhood of $\varphi_{1}$ in $W^{n, \infty}[-r, 0]$.

Next we consider a class of systems $\Lambda$ which are linear in $x$. As a relaxed system, $\Lambda$ is linear.

$\Lambda$

$$
\begin{aligned}
& \dot{x}(t)=L(t) x_{t}+b(v(t), t) \quad(t \in T), \\
& x_{t_{0}}=\varphi_{0},
\end{aligned}
$$

where $v \in \mathscr{S}^{\#}$ and

(2.6) for $L: T \rightarrow \mathscr{L}\left(C^{n}[-r, 0], \mathbb{R}^{n}\right)$ the map $t \mapsto L(t) \varphi$ is measurable for all $\varphi \in$ $C^{n}[-r, 0]$ and ess sup $\|L(t)\|<\infty$; the function $b: T \times \Omega_{0} \rightarrow \mathbb{R}^{n}$ is measurable in the first, continuous in the second argument, and satisfies

$$
\underset{t \in T}{\operatorname{ess} \sup } \sup _{\omega \in \Omega_{0}}|b(t, \omega)|<\infty
$$

furthermore, $\varphi_{0} \in C^{n}[-r, 0]$ and $t_{1}-r>t_{0}$.

For each $v \in \mathscr{S}^{\#}$, the system $\Lambda$ has a unique trajectory $x$ with $x_{t}=\varphi_{0}$, Hale [14, p. 142]. The system $\Lambda$ is a special case of $\Sigma$ satisfying conditions (1.1)-(1.4).

Remark 2.4. Consider a function $b: T \times \Omega_{0} \rightarrow \mathbb{R}^{n}$ with the same properties as in (2.6). Then the map $t \mapsto b(t, \cdot)$ may be considered as a map on $T$ with values in $C^{n}\left(\Omega_{0}\right)$. Hence, since $C^{n}\left(\Omega_{0}\right)$ is separable, $b$ may be identified with an element of $L_{\infty}\left(T, C^{n}\left(\Omega_{0}\right)\right)$ where $\|b\|_{\infty}:=\operatorname{ess}_{\sup } \sup _{\omega \in \Omega_{0}}|b(t, \omega)|$ (cf. Warga [27, p. 122 and Theorem I.5.26]).

Remark 2.5. The set of trajectories of $\Lambda$ coincides with the set of trajectories of the following system with ordinary controls:

$$
\dot{x}(t)=L(t) x_{t}+u(t) \quad(t \in T),
$$

where the controls $u$ are measurable functions defined on $T$ with values $u(t)$ in co $b(\Omega(t), t)$. Hence the reachability theories for these two classes of systems coincide. However, we write down the proofs for the system $\Lambda$, since the corresponding optimal control theories are different and the extra expense of writing is minimal. 
THEOREM 2.3. For the system $\Lambda$ a function $\varphi_{1}$ in $W^{n, \infty}[-r, 0]$, (respectively a point $\alpha$ in $\left.\mathbb{R}^{n}\right)$, is stably reachable iff it is locally reachable.

Proof. One direction is trivial. Let $N$ be a neighborhood of $\varphi_{1}$ in $W^{n, \infty}[-r, 0]$ such that each element of $N$ is reachable at time $t_{1}$. Suppose that $\varphi_{1}$ is reached with the trajectory $x^{0}$ of $\Lambda$ corresponding to $v^{0} \in \mathscr{S}^{\#}$. For $\varepsilon>0$, define a neighborhood $N^{\varepsilon}$ of $\varphi_{1}$ by

$$
N^{\varepsilon}:=\varphi_{1}+\varepsilon\left(N-\varphi_{1}\right) .
$$

Let $\varphi^{\varepsilon} \in N^{\varepsilon}$. Then there is $\varphi \in N$ with $\varphi^{\varepsilon}=\varphi_{1}+\varepsilon\left(\varphi-\varphi_{1}\right)$. The function $\varphi$ is reachable, say with $x$ corresponding to $v \in \mathscr{S}^{\#}$. Then by linearity, $\varphi^{\varepsilon}$ is reached with $x^{\varepsilon}:=x^{0}+$ $\varepsilon\left(x-x^{0}\right)$ corresponding to

$$
v^{\varepsilon}:=v^{0}+\varepsilon\left(v-v^{0}\right) \in \mathscr{S}^{\#} .
$$

For all $\delta>0$ there is $\varepsilon>0$ such that for all $\varphi^{\varepsilon} \in N^{\varepsilon}$ the trajectory $x^{\varepsilon}$ satisfies $\left\|x^{\varepsilon}-x^{0}\right\|_{\infty}<$ $\delta$, since the trajectories of $\Lambda$ are uniformly bounded. Thus $\varphi_{1}$ is reached stably with $x^{0}$. The assertion for $\alpha \in \mathbb{R}^{n}$ follows similarly.

Remark 2.6. Suppose that $\varphi_{1}$ is reached stably with $x^{0}$ and $x^{1}$ is any trajectory of $\Lambda$ reaching $\varphi_{1}$. Then the theorem above shows that $\varphi_{1}$ is reached stably with $x^{1}$. Hence for linear systems it is not necessary to specify the trajectory reaching $\varphi_{1}$ stably, if $\varphi_{1}$ is stably reachable with some trajectory.

Remark 2.7. For scalar systems (i.e. $n=1$ ) stable reachability of points in $\mathbb{R}$ follows under very mild assumptions. Suppose the trajectories of $\Sigma$ are uniformly bounded and there are $v^{0}, \bar{v}, \underline{v} \in \mathscr{S}^{\#}$ such that the map $v \mapsto x\left(v, t_{1}\right)$ has Gateaux derivatives at $v^{0}$ in the directions $\bar{v}-v^{0}$ and $\underline{v}-v^{0}$ satisfying

$$
D\left(x\left(t_{1}, v^{0}\right) ; \bar{v}-v^{0}\right)>0 \text { and } D\left(x\left(t_{1}, v^{0}\right) ; \underline{v}-v^{0}\right)<0 .
$$

Then $x\left(t_{1}, v^{0}\right)$ is stably reachable with $x\left(v^{0}\right)$, since the map $v \mapsto x\left(t_{1}, v\right)$ is continuous and $n=1$.

3. Regular and stable reachability. In this section, we introduce the concept of regular reachability and investigate its relation to local and stable reachability.

Definition 3.1. A function $\varphi_{1}$ in $W^{n, \infty}[-r, 0]$ is called regularly reachable at time $t_{1}$ with a trajectory $x^{0}$ of $\Sigma$, iff $\varphi_{1}(-r)=x^{0}\left(t_{1}-r\right)$ and there exists a neighborhood $V$ of $0 \in \mathbb{R}^{n}$ such that

$$
V \subset-\dot{\varphi}_{1}\left(t-t_{1}\right)+\operatorname{co} f\left(x_{t}^{0}, \Omega(t), t\right) \quad\left(t \in T_{1}\right) .
$$

A trajectory $x^{0}$ is called regular, iff $x_{t_{1}}^{0}$ is reached regularly at time $t_{1}$ with $x^{0}$; otherwise $x^{0}$ is called irregular. By Lemma 1.4 it follows-which is to be expected-that $\varphi_{1}=x_{t_{1}}^{0}$, if $x^{0}$ reaches $\varphi_{1}$ regularly. A function $\varphi_{1} \in W^{n, \infty}[-r, 0]$ is reached regularly with $x^{0}$ iff $\varphi_{1}(-r)=x^{0}\left(t_{1}-r\right)$ and in $L_{\infty}^{n}$

$$
\begin{aligned}
\dot{\varphi}_{1} \in \operatorname{int} & \left\{z \in L_{\infty}^{n}[-r, 0]: z\left(t-t_{1}\right) \in \operatorname{co} f\left(x_{t}^{0}, \Omega(t), t\right)\left(t \in T_{1}\right)\right\} \\
& =\operatorname{int}\left\{z \in L_{\infty}^{n}[-r, 0]: z\left(t-t_{1}\right)=f\left(x_{t}^{0}, v(t), t\right)\left(t \in T_{1}\right) \text { for a } v \in \mathscr{S}^{\#}\right\} .
\end{aligned}
$$

Regular reachability means that not only $\dot{\varphi}_{1}\left(t-t_{1}\right)$, but a whole uniform neighborhood of $\dot{\varphi}_{1}\left(t-t_{1}\right)$ is contained in the set of relaxed velocity vectors if the system at time $t$ is in the state $x_{t}^{0}$.

If the optimal trajectory $x^{0}$ is regular, the maximum principle constitutes a necessary optimality criterion (see Colonius [9], [10]).

The following theorem relates stable reachability of points in $\mathbb{R}^{n}$ and final states in $W^{n, \infty}[-r, 0]$ via the notion of regular reachability. 
THEOREM 3.1. Assume that $\Sigma$ satisfies (1.3) and that for $\varphi_{1} \in W^{n, \infty}[-r, 0]$ the following assumptions hold:

(a) the point $\varphi_{1}(-r)$ is reached stably with $x^{0}$ at $t_{1}-r$;

(b) the function $\varphi_{1}$ is reached regularly with $x^{0}$ at $t_{1}$. Then $\varphi_{1}$ is reached stably with $x^{0}$ at $t_{1}$.

Proof. By assumption (b) and the stability lemma 2.1, there are $\delta>0$ and a neighborhood $N$ of $\varphi_{1} \in W^{n, \infty}[-r, 0]$ such that for all $\varphi \in N$ and all $x$ with $\left\|x-x^{0}\right\|_{\infty}<\delta$

$$
\dot{\varphi}\left(t-t_{1}\right) \in \operatorname{cof} f\left(x_{t}, \Omega(t), t\right) \quad\left(t \in T_{1}\right) .
$$

By assumption (a), there is a neighborhood $N_{1}$ of $\varphi_{1} \in W^{n, \infty}[-r, 0]$, such that for all $\varphi \in N_{1}$ there is $x^{\varphi} \in C^{n}\left[t_{0}-r, t_{1}-r\right]$ with $\left\|x^{\varphi}-x^{0}\right\|_{\infty}<\delta$ and

$$
\dot{x}^{\varphi}(t)=f\left(x_{t}^{\varphi}, v^{\varphi}(t), t\right) \quad\left(t \in\left[t_{0}, t_{1}-r\right]\right)
$$

for a relaxed control $v^{\varphi} \in \mathscr{S}^{\#}$, and

$$
x_{t_{0}}^{\varphi}=\varphi_{0}, \quad x^{\varphi}\left(t_{1}-r\right)=\varphi(-r) .
$$

Without loss of generality, we may assume that $N_{1} \subset N$. The function $x^{\varphi}$ can be extended to an absolutely continuous function on $\left[t_{0}-r, t_{1}\right]$ by

$$
x_{t_{1}}^{\varphi}:=\varphi .
$$

It only remains to prove that $x^{\varphi}$ is a trajectory of $\Sigma$. We have to show that there is $v \in \mathscr{S}^{\#}$ such that

$$
\dot{x}^{\varphi}(t)=\dot{\varphi}\left(t-t_{1}\right)=f\left(x_{t}^{\varphi}, v(t), t\right) \quad\left(t \in T_{1}\right)
$$

or, equivalently,

$$
\dot{\varphi}\left(t-t_{1}\right) \in \operatorname{co} f\left(x_{t}^{\varphi}, \Omega(t), t\right) \quad\left(t \in T_{1}\right) .
$$

This follows by $N_{1} \subset N$ and $\left\|x^{\varphi}-x^{0}\right\|_{\infty}<\delta$.

COROllary 3.1. Assume that $\varphi_{1} \in W^{n, \infty}[-r, 0]$ is regularly reachable with $x^{0}$. Then for all $y$ in a neighborhood of $\dot{\varphi}_{1} \in L_{\infty}^{n}[-r, 0]$ there is a trajectory $x$ of $\Sigma$ with

$$
\dot{x}_{t_{1}}=y \text { and } x(t)=x^{0}(t) \text { for } t \in\left[t_{0}, t_{1}-r\right] \text {. }
$$

Proof. First reach $\varphi_{1}(-r)$ with $x^{0}$. Then use regularity as above in order to steer the system to

$$
\left(\varphi_{1}(-r)+\int_{-r}^{s} y(\tau) d \tau, s \in[-r, 0]\right) \in W^{n, \infty}[-r, 0]
$$

Theorem 3.1 shows that, up to a finite dimensional reachability condition at $t_{1}-r$, regular reachability implies stable reachability. We shall prove a partial converse of this for a special class of systems.

Define the class of systems $\Sigma_{a}$, where the control term appears addititively, by:

$$
\begin{aligned}
& \dot{x}(t)=a\left(x_{t}, t\right)+b(v(t), t) \quad(t \in T), \\
& x_{t_{0}}=\varphi_{0},
\end{aligned}
$$

where $v \in \mathscr{S}^{\#}$ and, in addition to the requirements of (2.6), (3.2) the map $a: C^{n}[-r, 0] \times$ $T \rightarrow \mathbb{R}^{n}$ is continuous in the first and measurable in the second argument, and $|a(\varphi, t)|$ is essentially bounded for bounded arguments. 
$\Sigma_{a}$ is a special case of $\Sigma$ satisfying (1.1) and (1.2). Furthermore, we assume unique solvability, i.e., (1.4).

Remark 3.1. As in Remark 2.5, the set of trajectories of $\Sigma_{a}$ coincides with the set of trajectories of

$$
\dot{x}(t)=a\left(x_{t}, t\right)+u(t),
$$

where the controls $u$ take values $u(t)$ in co $b(\Omega(t), t)$.

We shall make use of the following generalization of a classical result due to A. Denjoy.

THEOREM 3.2. Let $X$ be a complete separable metric space, $S$ a compact metric space, $h:[a, b] \times S \rightarrow X$ such that $h(\cdot, s)$ is measurable and $h(t, \cdot)$ is continuous for all $(t, s) \in[a, b] \times S$. Then for a subset $T^{\prime}$ of $[a, b]$ with $\lambda\left(T^{\prime}\right)=\lambda([a, b])$ and for all $t \in T^{\prime}$ there is a set $E \subset[a, b]$ such that $t$ is a point of density of $E$ and $h \mid E \times S$ is continuous in $t$.

Proof. By a strong version of Lusin's theorem (see Warga [27, Thm. I.5.26(2)]), there is for all $\varepsilon>0$ a closed subset $F_{\varepsilon}$ of $[a, b]$ such that $\lambda\left([a, b] \mid F_{\varepsilon}\right) \leqq \varepsilon$ and $h \mid F_{\varepsilon} \times S$ is continuous. This implies the theorem above in exactly the same way as the usual Lusin's theorem implies Natanson [21, Satz 2, p. 296].

THEOREM 3.3. Assume that the trajectories of $\Sigma_{a}$ are uniformly bounded, and that there is a neighborhood $U$ of $z^{0} \in L_{\infty}^{n}\left(T_{1}\right)$ such that for all $z \in U$ there is a trajectory $x$ with $z=(\dot{x})_{t_{1}}$. Then

$$
\operatorname{int}\left\{z \in L_{\infty}^{n}\left(T_{1}\right): z(t) \in \operatorname{co} b(\Omega(t), t)\left(t \in T_{1}\right)\right\} \neq \varnothing .
$$

Proof. There is a trajectory $x^{0}$ with $z^{0}=\left(\dot{x}^{0}\right)_{t_{1}}$. Define for $t \in T_{1}$

$$
V_{t}:=-z^{0}(t)+a\left(x_{t}^{0}, t\right)+\operatorname{co} b(\Omega(t), t) .
$$

Then $V_{t}$ is convex and $0 \in V_{t}$. Due to the assumptions on $\Sigma_{a}$, all sets $V_{t}$ are contained in a compact set $C \subset \mathbb{R}^{n}$. Consider $\Phi: t \mapsto V_{t}$ as a map from $T_{1}$ into the set of closed subsets of $C$. Then $\Phi$ is well defined and measurable by Warga [27, Thm. I.7.6]. Thus by Warga [27, Thm. I.7.8], there is a countable set $\left\{\xi_{i}: i \in \mathbb{N}\right\}$ of measurable selections of $\Phi$ such that

$$
\left\{\xi_{i}(t): i \in \mathbb{N}\right\} \text { is dense in } \Phi(t)=V_{t} \text { for a.a. } t \in T_{1} .
$$

For $y \in \mathscr{E}:=\left\{y \in \mathbb{R}^{n}:|y|=1\right\}$ and $t \in T_{1}$ let

$$
\bar{h}(y, t):=\sup \left\{y \xi_{i}(t): i \in \mathbb{N}\right\}, \quad \underline{h}(y, t):=\inf \left\{y \xi_{i}(t): i \in \mathbb{N}\right\} .
$$

Then $\bar{h}$ and $\underline{h}$ are continuous in $y$, measurable in $t$ and satisfy

$$
\bar{h}(y, t)=\max \left\{y \xi: \xi \in V_{t}\right\}, \quad \underline{h}(y, t)=\min \left\{y \xi: \xi \in V_{t}\right\} .
$$

We shall prove that there are a constant $\alpha>0$ and a set $T^{\prime} \subset T_{1}$ with $\lambda\left(T^{\prime}\right)=\lambda\left(T_{1}\right)$ such that for all $t \in T^{\prime}$ and all $y \in \mathscr{E}$

$$
\bar{h}(y, t) \geqq \underline{h}(y, t)+\alpha .
$$

Then, by Warga [27, Thm. I.7.10], there exists a measurable function $\xi: T_{1} \rightarrow C$ with $\xi(t) \in V_{t}, t \in T^{\prime}$ such that for all $y \in \mathscr{E}$ and all $t \in T^{\prime}$

$$
\min \left\{y \xi: \xi \in V_{t}\right\}+\frac{\alpha}{2} \leqq y \xi(t) \leqq \max \left\{y \xi: \xi \in V_{t}\right\}-\frac{\alpha}{2} .
$$

Then $\xi \in \operatorname{int}\left\{z \in L_{\infty}^{n}\left(T_{1}\right): z(t) \in V_{t}\left(t \in T_{1}\right)\right\}$, and the theorem follows. 
The set $\mathscr{E}$ and the set $\mathscr{T}$ of trajectories of $\Sigma_{a}$ are compact metric spaces, since $\mathscr{T}$ is compact in $C^{n}\left[t_{0}-r, t_{1}\right]$ by Lemma 1.3. Consider $a$ as a map: $\mathscr{T} \times T_{1} \rightarrow \mathbb{R}^{n}$, $(x, t) \mapsto a\left(x_{t}, t\right)$. Then $a$ is continuous in $x$ and measurable in $t$.

Now Theorem 3.2 implies that there is $T^{\prime} \subset T_{1}$ with $\lambda\left(T^{\prime}\right)=\lambda(T)$ such that for all $t^{\prime} \in T^{\prime}$, all $y \in \mathscr{E}$, and all $x \in \mathscr{T} \bar{h}(y, t), \underline{h}(y, t)$, and $a\left(x_{t}, t\right)$ are approximately continuous in $t=t^{\prime}$. There exists a constant $\alpha>0$ with the following property: For any $t^{\prime} \in T^{\prime}$ and any $y \in \mathscr{E}$, there is a step function $z$ such that $z^{0}+z \in U$ and that $y z(t)$ has a jump of length greater than $2 \alpha$ in $t=t^{\prime}$.

For any such $z$ there exists a solution $(x, v)$ of $\Sigma_{a}$ with

$$
z(t)=-z^{0}(t)+a\left(x_{t}, t\right)+b(v(t), t) \quad\left(t \in T_{1}\right) .
$$

Hence

$$
y z(t)=y\left[a\left(x_{t}, t\right)-a\left(x_{t}^{0}, t\right)\right]+y\left[-z^{0}(t)+a\left(x_{t}^{0}, t\right)+b(v(t), t)\right] \quad\left(t \in T_{1}\right) .
$$

The left-hand side has a jump of length greater than $2 \alpha$ in $t=t^{\prime}$ and $y\left[a\left(x_{t}, t\right)-a\left(x_{t}^{0}, t\right)\right]$ is approximately continuous in $t=t^{\prime}$. Hence (3.3) follows.

Remark 3.2. The proof above owes a lot to the proof of Schwarzkopf [24, Thm. 1].

Remark 3.3. Let $\varphi_{1} \in W^{n, \infty}[-r, 0]$. Then the assumptions of Theorem 3.3 are satisfied for $z^{0}(t)=\dot{\varphi}_{1}\left(t-t_{1}\right), t \in T_{1}$, if $\varphi_{1}$ is reached locally (resp. stably) with $x^{0}$ at $t_{1}$, and the trajectories of $\Sigma_{a}$ are uniformly bounded. Then it also follows that $\varphi_{1}(-r)$ is reached locally (resp. stably) with $x^{0}$ at $t_{1}-r$. If one could prove that $V_{t}$ contains a neighborhood of $0 \in \mathbb{R}^{n}$ which is independent of $t \in T_{1}$, the converse of Theorem 3.1 would hold. However, in the following we give an example of a linear system where a certain final state $\varphi_{1}$ is locally reachable (i.e., by Theorem 2.3 also stably reachable), but reached irregularly with a certain trajectory $x^{0}$. Hence in general, we cannot show more than Theorem 3.3. Intuitively, this may be explained as follows:

In the proof of Theorem 3.1, we reach $\varphi_{1}$ with $x^{0}$. In order to reach all $\varphi$ in a neighborhood of $\varphi_{1} \in W^{n, \infty}[-r, 0]$, we reach first $\varphi(-r)$; by stable reachability of $\varphi_{1}(-r)$, we can achieve this with small deviations from $x^{0} \mid\left[t_{0}, t_{1}-r\right]$. The hereditary effects influencing the possible velocity vectors of the system on $T_{1}$ are compensated using regular reachability of $\varphi_{1}$ : By the stability lemma 2.1, regularity allows to choose controls in such a way that arbitrary small deviations from $x^{0}$ are compensated and all velocity vectors $\dot{\varphi}\left(t-t_{1}\right)$ in a uniform neighborhood of $\dot{\varphi}_{1}\left(t-t_{1}\right)$ are reached.

Now one can try to reach $\varphi(-r)$ in such a way that the hereditary effects influencing the system behavior on $T_{1}$ prepare the reaching of $\varphi$. Then it is not necessary to compensate arbitrary small deviations from $x^{0}$ on $\left[t_{0}, t_{1}-r\right]$ and we may get local reachability without regularity. This is performed in the following scalar example. Theorem 3.3 shows the limitations of this manipulation: One can achieve a shift of the set of velocity vectors, but $V_{t}$ must contain interior points.

Example 3.1. Consider

$$
\begin{aligned}
& \dot{x}(t)=x(t-2)+u(t) \quad(t \in T:=[0,6]), \\
& x_{0}=0, \\
& \Omega:=[-3,1] .
\end{aligned}
$$

We claim that

$$
\varphi_{1}(t):= \begin{cases}t+2, & t \in[-2,-1] \\ 1, & t \in[-1,2]\end{cases}
$$


is locally and irregularly reachable. Define an (ordinary) control function $u^{1}$ by

$$
u^{1}(t):= \begin{cases}0 & \text { for } t \in[0,4] \\ 1 & \text { for } t \in(4,5], \\ 0 & \text { for } t \in(5,6]\end{cases}
$$

The corresponding trajectory $x^{1}$ is given by

$$
x^{1}(t):= \begin{cases}0 & \text { for } t \in[-2,4] \\ t-4 & \text { for } t \in(4,5), \\ 1 & \text { for } t \in[5,6]\end{cases}
$$

Clearly $x^{1}$ is irregular and satisfies $x_{6}^{1}=\varphi_{1}$. Now we shall show that the assumptions of Theorem 2.2 are satisfied for a certain trajectory $x^{0}$. This will imply that $\varphi_{1}$ is locally reachable.

Define a control function $u^{0}$ by

$$
u^{0}(t)= \begin{cases}1 & \text { for } t \in[0,2), \\ -2 & \text { for } t \in[2,4), \\ -\frac{1}{2}(t-4)^{2}+2(t-2)-5 & \text { for } t \in[4,5), \\ -\frac{1}{2}(t-4)^{2}+2(t-2)-6 & \text { for } t \in[5,6]\end{cases}
$$

The corresponding trajectory $x^{0}$ is given by

$$
x^{0}(t)= \begin{cases}0 & \text { for } t \in[-2,0], \\ t & \text { for } t \in[0,2], \\ \frac{1}{2}(t-2)^{2}-2 t+6 & \text { for } t \in[2,4] \\ t-4 & \text { for } t \in[4,5] \\ 1 & \text { for } t \in[5,6]\end{cases}
$$

Clearly $x_{6}^{0}=\varphi_{1}$.

An elementary analysis shows that $u^{0}(t)$ is uniformly in the interior of $\Omega$ on $[2,6]$; i.e., the assumptions of Theorem 2.2 are satisfied.

Assume that (1.3) holds. Then the system $\Sigma$ can be linearized, and the linearized system $\Sigma_{\text {lin }}$ has the form (1.5), (1.6) as indicated in Lemma 1.5.

Regular reachability has the remarkable property, that it is invariant under linearization. More precisely, we have

PROPOSITION 3.1. Suppose that (1.3) holds, and $\varphi_{1}=x_{t_{1}}^{0}$, where $x^{0}$ is the trajectory of $\Sigma$ corresponding to $v^{0} \in \mathscr{S}^{\#}$. Then $x^{0}$ reaches $\varphi_{1}$ regularly iff the zero trajectory of $\Sigma_{\operatorname{lin}}$ reaches $0 \in W^{n, \infty}[-r, 0]$ regularly.

Proof. Write down the definitions!

This property motivates interest in regular reachability of $0 \in W^{n, \infty}[-r, 0]$ with the zero trajectory. Consider the following linear system:

$$
\begin{aligned}
& \dot{x}(t)=L(t) x_{t}+B(t) v(t) \quad(t \in T), \\
& x_{t_{0}}=0,
\end{aligned}
$$

where we assume that the assumptions in (2.6) are satisfied and $B \in L_{\infty}\left(T, \mathbb{R}^{n \times m}\right)$.

Corollary 3.2. Let $0 \in$ int $_{\delta} \operatorname{co} \Omega(t)\left(t \in T_{1}\right)$ for $a \delta>0$. Then the following two conditions on the system (3.4), (3.5) are equivalent:

(i) The trajectory $x^{0}=0$ reaches $0 \in W^{n, \infty}[-r, 0]$ regularly at time $t_{1}$;

(ii) Rank $B(t)=n\left(t \in T_{1}\right)$ and $B(t)^{+}$is essentially bounded on $T_{1}$. 
Furthermore, $0 \in W^{n, \infty}[-r, 0]$ is locally reachable at time $t_{1}$, iff (ii) holds and $0 \in \mathbb{R}^{n}$ is locally reachable at time $t_{1}-r$.

Proof. Define the multiplication operator $\tilde{B}: L_{\infty}^{m}\left(T_{1}\right) \rightarrow L_{\infty}^{n}\left(T_{1}\right)$ by

$$
(\tilde{B} u)(t)=B(t) u(t) \quad\left(t \in T_{1}\right) .
$$

By Kurcyusz/Olbrot [17, Lemma 3], $\tilde{B}$ has a closed image iff $B(t)^{+}$is essentially bounded on $T_{1}$. Hence $B(t)^{+}$is essentially bounded and rank $B(t)=n\left(t \in T_{1}\right)$ iff the linear map $B$ is surjective, hence open. Because of $0 \in$ int $_{\delta} \operatorname{co} \Omega(t)$, this in turn is equivalent to the existence of a neighborhood $V$ of $0 \in \mathbb{R}^{n}$ such that

$$
V \subset V_{t}=\cos B(t) \Omega(t)=B(t) \cos \Omega(t) \quad\left(t \in T_{1}\right) .
$$

This proves the equivalence of (i) and (ii). If (i) holds and $0 \in \mathbb{R}^{n}$ is locally reachable at $t_{1}-r$, then $0 \in W^{n, \infty}[-r, 0]$ is locally reachable by Theorem 2.3 and Theorem 3.1.

The converse follows by Theorem 3.3 and the assumption

$$
0 \in \text { int }_{\delta} \operatorname{co} \Omega(t)\left(t \in T_{1}\right) .
$$

Remark 3.4. The last equivalence in the corollary above is the analogue of results in the theory of unconstrained linear hereditary systems. Here the following is known (for controls $u \in L_{p}^{m}(T), 1 \leqq p \leqq \infty$ ):

Suppose that $B(t)^{+}$is essentially bounded on $T_{1}$. Then each element $\varphi \in$ $W^{n, p}[-r, 0]$ is reachable at time $t_{1}$, iff each element $\alpha \in \mathbb{R}^{n}$ is reachable at time $t_{1}-r$ and rank $B(t)=n\left(t \in T_{1}\right)$ (see Jacobs/Kao [15], Banks/Jacobs/Langenhop [2], [3], Kurcyusz/Olbrot [17], Colonius/Hinrichsen [11]). In fact, by a category argument, Corollary 3.2 implies the result above for $p=\infty$. Furthermore, complete reachability in $W^{n, \infty}$ implies even that $B(t)^{+}$is essentially bounded on $T_{1}$. This proves a conjecture by Banks/Jacobs/Langenhop [3] (see also Kurcyusz/Olbrot [17, p. 48] and Colonius/Hinrichsen $[11$, p. 878]). In turn the cited result implies the last assertion of Corollary 3.2, provided that $B(t)^{+}$is essentially bounded on $T_{1}$ as shown in Colonius [9, Remark 10].

Remark 3.5. If $B(t)$ depends continuously on $t$ and has a constant rank, then by Kurcyusz/Olbrot [17, Lemma 4] the generalized inverse $B(t)^{+}$is bounded.

Remark 3.6. Suppose there is $\varepsilon>0$ such that for a.a. $t \in\left[t_{1}-r-\varepsilon, t_{1}-r\right]$ one has $0 \in$ int $_{\delta} \operatorname{co} \Omega(t)$ for a $\delta>0$, and the generalized inverse $B(t)^{+}$is essentially bounded and has rank $n$. Then by Theorem $2.20 \in \mathbb{R}^{n}$ is locally reachable at time $t_{1}-r$.

4. Analysis of the reachable set $\mathscr{R}$ of final states and of the set $\mathscr{T}$ of trajectories. In $\S 3$, we analyzed reachability properties of a specific final state $\varphi_{1}$ and of a specific trajectory $x^{0}$. Now we change our point of view by analyzing properties of the whole set $\mathscr{T}$ of trajectories:

$$
\mathscr{T}:=\left\{x \in C^{n}\left[t_{0}-r, t_{1}\right]: x \text { is a trajectory of } \Sigma\right\}
$$

and of the whole reachable set $\mathscr{R}$ of final states

$$
\mathscr{R}:=\left\{\varphi \in W^{n, \infty}[-r, 0]: \text { there is } x \in \mathscr{T} \text { with } \varphi=x_{t_{1}}\right\} .
$$

Since the initial state $\varphi_{0}$ remains fixed throughout, we may identify $x \in \mathscr{T}$ with $x \mid T$ and consider $\mathscr{T}$ as a subset of $W^{n, \infty}(T)$. The set $\mathscr{R}$ will always be considered in $W^{n, \infty}$-topology.

Observe that by definition $\varphi \in$ int $\mathscr{R}$ iff $\varphi$ is locally reachable.

LEMMA 4.1. Consider the system $\Sigma_{a}$ of $\S 3$, and assume that $x^{0}, x^{1} \in \mathscr{T}$ correspond to $v^{0}, v^{1} \in \mathscr{S}^{\#}$ and that $x^{1}$ is regular. Then the trajectories $x^{\gamma} \in \mathscr{T}$ corresponding to $v^{\gamma}:=(1-\gamma) v^{0}+\gamma v^{1} \in \mathscr{S}^{\#}$ where $0<\gamma \leqq 1$ are regular. 
Proof. By regularity of $x^{1}$ there is $\delta>0$ such that

$$
\dot{x}^{1}(t)-a\left(x_{t}^{1}, t\right)=b\left(v^{1}(t), t\right) \in \text { int }_{\delta} \operatorname{co} b(\Omega(t), t) \quad\left(t \in T_{1}\right) .
$$

Since $b\left(v^{0}(t), t\right) \in \operatorname{co} b(\Omega(t), t) \quad\left(t \in T_{1}\right)$ and $\operatorname{co} b(\Omega(t), t)$ is convex, this implies for $0<\gamma \leqq 1$

$$
\begin{aligned}
\dot{x}^{\gamma}(t)-a\left(x_{t}^{\gamma}, t\right)=b\left(v^{\gamma}(t), t\right) & =(1-\gamma) b\left(v^{0}(t), t\right)+\gamma b\left(v^{1}(t), t\right) \\
& \in \text { int }_{\gamma \delta} \operatorname{co} b(\Omega(t), t) \quad\left(t \in T_{1}\right) .
\end{aligned}
$$

This shows regularity of $x^{\gamma}$.

Remark 4.1. Using the same construction as in the proof above, one can see that for $\Sigma_{a}$ the set $\mathscr{T}$ is path connected, i.e., any two trajectories $x^{0}, x^{1} \in \mathscr{T}$ can be connected by a continuous path $\left(x^{\gamma}, \gamma \in[0,1]\right)$. One only has to prove that the map $v^{\gamma} \rightarrow x^{\gamma}: \mathscr{S}^{\#} \rightarrow W^{n, \infty}(T)$ is continuous with respect to norm topology on $W^{n, \infty}(T)$ and strong norm topology on $\mathscr{S}^{\#}$. This follows by Lemma 1.3 and Lemma 1.2.

Define the finite dimensional reachable set $\mathscr{R}^{f}$ at time $t_{1}-r$ as

$$
\mathscr{R}^{f}:=\left\{\alpha \in \mathbb{R}^{n}: \text { there is } \varphi \in \mathscr{R} \text { with } \alpha=\varphi(-r)\right\},
$$

and let

$$
\mathscr{R}_{\alpha}:=\{\varphi \in \mathscr{R}: \varphi(-r)=\alpha\} \text { for } \alpha \in \mathscr{R}^{f}
$$

Then

$$
\mathscr{R}=\bigcup_{\alpha \in \mathscr{R}^{f}} \mathscr{R} \alpha
$$

is a decomposition of the reachable set parametrized by the elements of the finite dimensional reachable set at time $t_{1}-r$. We identify $\mathscr{R}_{\alpha}$ with a subset of $L_{\infty}^{n}[-r, 0]$, and consider $\mathscr{R}_{\alpha}$ in the induced topology. Then

$$
\text { int } \begin{aligned}
\mathscr{R}_{\alpha}=\left\{\varphi_{1} \in \mathscr{R}_{\alpha}: \text { there is a } \delta>0 \text { such that all } \varphi \in W^{n, \infty}[-r, 0]\right. \\
\text { with } \left.\varphi(-r)=\alpha \text { and }\left\|\dot{\varphi}_{1}-\dot{\varphi}_{\infty}\right\|<\delta \text { are in } \mathscr{R}_{\alpha}\right\} .
\end{aligned}
$$

Corollary 3.1 shows that each regularly reachable $\varphi_{1}$ lies in int $\mathscr{R}_{\varphi_{1}(-r)}$.

THEOREM 4.1. Assume that $\Sigma_{a}$ satisfies (1.3). Then

(i) there exists a regularly reachable final state $\varphi_{1}$ in $\mathscr{R}_{\alpha}$ for each $\alpha \in \mathscr{R}^{f}$ iff

$$
\text { int }_{\delta} \operatorname{co} b(\Omega(t), t) \neq \varnothing \quad\left(t \in T_{1}\right) \quad \text { for } a \delta>0 .
$$

(ii) Suppose (4.1) holds. Then the set of regularly reachable final states is open and dense in $\mathscr{R}_{\alpha}$ for each $\alpha \in \mathscr{R}^{f}$.

(iii) Suppose that $\varphi_{1}$ is reached regularly and stably with a certain trajectory $x^{0}$. Then there is a neighborhood $N$ of $\varphi_{1}$ in $W^{n, \infty}[-r, 0]$ such that all $\varphi \in N$ are regularly reachable.

Proof. (i) One direction is trivial. Suppose (4.1) holds and let $z^{0} \in L_{\infty}^{n}\left(T_{1}\right)$ with

$$
z^{0}(t) \in \text { int }_{\delta} \operatorname{co} b(\Omega(t), t) \quad\left(t \in T_{1}\right) .
$$

For any trajectory $x^{0}$ define $x^{1}$ by

$$
x^{1}(t):=x^{0}(t), \quad t \in\left[t_{0}-r, t_{1}-r\right], \quad \dot{x}^{1}(t)=a\left(x_{t}^{1}, t\right)+z^{0}(t) \quad\left(t \in T_{1}\right) .
$$

Then $x^{1}$ is a regular trajectory and $\varphi_{1}:=x_{t_{1}}^{1} \in \mathscr{R}_{x^{0}\left(t_{1}-r\right) \text {. }}$.

(ii) Let $\varphi_{1} \in \mathscr{R}$ be reached with the trajectory $x^{0}$ corresponding to $v^{0} \in \mathscr{S}^{\#}$. By

(i) there exists $\varphi_{2} \in \mathscr{R}_{\varphi_{1}(-r)}$ reached with a regular trajectory $x^{2}$ corresponding to 
$v^{2} \in \mathscr{S}^{\#}$. Define $x^{1}$ as the trajectory corresponding to the control $v^{1} \in \mathscr{S}^{\#}$ which coincides with $v^{0}$ on $\left[t_{0}, t_{1}-r\right)$ and with $v^{2}$ on $T_{1}$. Then $x^{1}\left(t_{1}-r\right)=\varphi_{1}(-r)$ and Lemma 4.1 together with Lemma 1.2 implies that in each neighborhood of $\varphi_{1}$ in $\mathscr{R}_{\varphi_{1}(-r)}$ there is a regularly reachable final state.

The set of regularly reachable final states is open in $\mathscr{R}_{\alpha}$ for $\alpha \in \mathscr{R}^{f}$ by the stability lemma 2.1.

(iii) The assumptions imply that there is $\delta>0$ such that

$$
\dot{\varphi}_{1}\left(t-t_{1}\right)-a\left(x_{t}^{0}, t\right) \in \text { int }_{3 \delta} \operatorname{co} b(\Omega(t), t) \quad\left(t \in T_{1}\right) .
$$

Furthermore there is a neighborhood $N$ of $\varphi_{1}$ in $W^{n, \infty}[-r, 0]$, such that all $\varphi \in N$ are reached with trajectories $x^{\varphi}$ satisfying

$$
\underset{t \in T_{1}}{\operatorname{ess} \sup _{1}}\left|a\left(x_{t}^{\varphi}, t\right)-a\left(x_{t}^{0}, t\right)\right|<\delta .
$$

If we choose $N$ so small that $\left\|\varphi-\varphi_{1}\right\|<\delta$ for all $\varphi \in N$, we find

$$
\dot{\varphi}\left(t-t_{1}\right)-a\left(x_{t}^{\varphi}, t\right) \in \text { int }_{\delta} \operatorname{co} b(\Omega(t), t) \quad\left(t \in T_{1}\right) .
$$

Corollary 4.1. Suppose that $\Sigma_{a}$ satisfies (1.3). Then in $L_{\infty}^{n} \operatorname{int}\left\{\dot{\varphi} \in L_{\infty}^{n}\left(T_{1}\right): \varphi \in\right.$ $\mathscr{R}\} \neq \varnothing$ iff (4.1) holds.

Proof. If condition (4.1) is satisfied, then by Theorem 4.1 there exists a regularly reachable final state $\varphi_{1}$. By Corollary 3.1 we find $\dot{\varphi}_{1} \in \operatorname{int}\left\{\dot{\varphi} \in L_{\infty}^{n}\left(T_{1}\right): \varphi \in \mathscr{R}\right\}$. The converse follows by Theorem 3.3.

Remark 4.2. If (4.1) holds a.e. on $\left[t_{1}-r-\varepsilon, t_{1}\right]$ for an $\varepsilon>0$, then by Theorem 2.2 it follows that int $\mathscr{R} \neq \varnothing$.

Remark 4.3. If the function $b$ does not depend explicitly on $t$ and $\Omega(t)=\Omega_{0}$ $(t \in T)$, then the condition (4.1) (nonempty interior in $L_{\infty}$-norm) reduces to int $\operatorname{co} b\left(\Omega_{0}\right) \neq \varnothing$. Furthermore, by the remark above and Corollary 4.1 , this condition is equivalent to int $\mathscr{R} \neq \varnothing$.

Remark 4.4. By Remark 2.4, functions $b$ satisfying the requirements in (2.6) may be considered as elements in $L_{\infty}\left(T, C^{n}\left(\Omega_{0}\right)\right)$. Now suppose that for a.a. $t \in T$ the set $\Omega(t)$ contains at least $n+1$ points. Then the set of functions $b$ satisfying condition (4.1) is open and dense in $L_{\infty}\left(T, C^{n}\left(\Omega_{0}\right)\right)$, and in this sense condition (4.1) is generic. Openness is easily seen. Density can be proved using a construction similar to that in the proof of Lemma 4.1: There exist $n+1$ measurable functions $\omega_{i}: T \rightarrow \Omega_{0}, i=$ $0,1, \cdots, n$ with $\omega_{i}(t) \in \Omega(t)$ and $\omega_{i}(t) \neq \omega_{j}(t)$ for a.a. $t \in T$ and $i \neq j$. Then for a.a. $t \in T$ there exists $b_{1}(t, \cdot) \in C^{n}\left(\Omega_{0}\right)$ mapping $\omega_{0}(t), \cdots, \omega_{n}(t)$ into $n+1$ points in $\mathbb{R}^{n}$ being in general position, i.e., having the property int $\cos _{\delta}\left\{b_{1}\left(t, \omega_{0}(t)\right), \cdots, b_{1}\left(t, \omega_{n}(t)\right)\right\} \neq \varnothing$ for some $\delta>0$. We may assume that $\delta$ is independent of $t$ and that $b_{1} \in L_{\infty}\left(T, C^{n}\left(\Omega_{0}\right)\right)$. Let $b_{0}$ be an arbitrary element of $L_{\infty}\left(T, C^{n}\left(\Omega_{0}\right)\right)$, and define

$$
b^{\gamma}(t, \omega):=\gamma b_{1}(t, \omega)+(1-\gamma) b_{0}(t, \omega) \text { for } t \in T, \omega \in \Omega_{0} .
$$

Then $b^{\gamma} \rightarrow b_{0}$ in $L_{\infty}\left(T, C^{n}\left(\Omega_{0}\right)\right)$ and

$$
\text { int }_{\gamma \delta} \operatorname{co}\left\{b \gamma\left(t, \omega_{0}(t)\right), \cdots, b^{\gamma}\left(t, \omega_{n}(t)\right)\right\} \neq \varnothing,
$$

i.e., the functions $b \gamma$ satisfy condition (4.1).

Remark 4.5. Theorem 4.1 (ii) shows that regular reachability is generic in $\mathscr{R}_{\alpha}$. Part (iii) shows regular reachability is preserved under small perturbations of $\varphi_{1}$, provided that it is also stable.

For linear systems, we can completely characterize regular reachability by strengthening the properties (ii) and (iii) in Theorem 4.1 . 
Corollary 4.2. Suppose that for the linear system $\Lambda$ condition (4.1) is satisfied. Then $\varphi_{1} \in W^{n, \infty}[-r, 0]$ is regularly reachable iff $\varphi_{1} \in$ int $\mathscr{R}_{\varphi_{1}(-r)}$. Furthermore, each element of int $\mathscr{R}$ is regularly reachable.

Proof. In view of Corollary 3.1 we have to show that each $\varphi_{1} \in \operatorname{int} \mathscr{R}_{\varphi_{1}(-r)}$ is regularly reachable. By Theorem 4.1(ii) there is $\varphi=W^{n, \infty}[-r, 0]$ such that $\varphi_{1}+\varphi \in$ $\mathscr{R}_{\varphi_{1}(-r)}$ is regularly reachable, say with $x^{0}$, and $\varphi_{1}-\varphi \in \mathscr{R}_{\varphi_{1}(-r)}$ is reachable, say with $x^{1}$. By linearity and Lemma 4.1 ,

$$
\varphi_{1}=\frac{1}{2}\left(\varphi_{1}+\varphi\right)+\frac{1}{2}\left(\varphi_{1}-\varphi\right)
$$

is reached regularly with $\frac{1}{2} x^{0}+\frac{1}{2} x^{1}$. Furthermore for each $\varphi \in$ int $\mathscr{R}$ we have $\varphi \in$ int $\mathscr{R}_{\varphi(-r)}$. This implies the last assertion.

Remark 4.6. It follows from Corollary 4.2 that each final state, which is reachable only with irregular trajectories lies in $\partial \mathscr{R}$ and clearly

$$
\bigcup_{\alpha \in \mathscr{R}^{f}} \partial \mathscr{R}_{\alpha} \subset \partial \mathscr{R} .
$$

However, there are regularly reachable final states in $\partial \mathscr{R} \bigcup_{\alpha \in \mathscr{R}^{f}} \partial \mathscr{R}_{\alpha}$ : For instance, reach $\alpha \in \partial \mathscr{R}^{f}$ with a trajectory $x^{0}$ satisfying $\dot{x}^{0}(t)-L(t) x_{t}^{0} \in \operatorname{int}_{\delta} \operatorname{co} b(\Omega(t), t)\left(t \in T_{1}\right)$ for a $\delta>0$. Then $x_{t_{1}}^{0} \in \partial \mathscr{R} \backslash \partial \mathscr{R}_{\alpha}$.

Regular reachability of a final function $\varphi_{1}$ means that there exists a certain trajectory reaching $\varphi_{1}$ regularly. Now in an optimization problem, we are interested in a specific unknown trajectory, the optimal one, out of all trajectories reaching $\varphi_{1}$. Thus regular reachability of $\varphi_{1}$ is only the minimal degree of well-posedness we have to require in an optimal control problem. In the following, we go a step further by asking: What can be said about regularity of all trajectories reaching a given final function $\varphi_{1}$ ?

Consider the system $\Sigma_{a}$. Here the regularity condition for $x^{0}$ is satisfied iff there is a neighborhood $V$ of $0 \in \mathbb{R}^{n}$ such that

$$
V \subset-\dot{\varphi}_{1}\left(t-t_{1}\right)+a\left(x_{t}^{0}, t\right)+\operatorname{co} b(\Omega(t), t) \quad\left(t \in T_{1}\right) .
$$

Now suppose that we can specify a uniform bound $c_{0}$ for $\mid a\left(x_{t}^{0}, t \mid, t \in T_{1}\right.$. If $\operatorname{co} b(\Omega(t), t)$ can be made big enough compared to $c_{0}$, all trajectories reaching $\varphi_{1}$ are regular. We give two examples where this idea applies.

Example 4.1.

$$
\begin{aligned}
& \dot{x}(t)=a\left(x_{t}, t\right)+v(t) \quad(t \in T), \\
& x_{t_{0}}=\varphi_{0}, \quad x_{t_{1}}=\varphi_{1},
\end{aligned}
$$

where $a$ is as in the definition of $\Sigma_{a}$, and additionally, satisfies

$$
\left|a\left(x_{t}, t\right)\right| \leqq 1 \quad\left(t \in T_{1}\right)
$$

for all trajectories $x$ reaching $\varphi_{1} . x$ is regular, iff there is a neighborhood $V$ of $0 \in \mathbb{R}^{n}$ such that

$$
V+\dot{\varphi}_{1}\left(t-t_{1}\right)-a\left(x_{t}, t\right) \subset \operatorname{int}_{\delta} \operatorname{co} \Omega(t) \quad\left(t \in T_{1}\right) .
$$

for a $\delta>0$.

If, e.g., $\varphi_{1}=0$, take $\Omega$ as a $n$-simplex with vertices $\omega_{0}, \omega_{1}, \cdots, \omega_{n}$ containing the unit ball of $\mathbb{R}^{n}$ in its interior. Then, obviously, all trajectories reaching $\varphi_{1}$ are regular. Observe that by Corollary $4.2 \mathscr{R}_{\varphi_{1}(-r)}$ is open. 
Example 4.2.

$$
\begin{aligned}
& \dot{x}(t)=A_{1} x(t-1)+v(t) \quad(t \in T:=[0, k+1]), \\
& x_{0}=\varphi_{0}, \quad x_{k+1}=\varphi_{1},
\end{aligned}
$$

where $\varphi_{0}, \varphi_{1} \in C^{n}[-1,0], k \in \mathbb{N}, A_{1} \in M_{n n}, \Omega:=\left\{\omega \in \mathbb{R}^{n}:|\omega| \leqq q\right\}$ and $q>0$. Then for $t \in[k, k+1]$

$$
\begin{aligned}
\left|A_{1} x(t-1)\right| \leqq\left\|A _ { 1 } \left|\left\|\mid x_{k}\right\|_{\infty}\right.\right. & \leqq\left\|A_{1}\right\|\left\{\left(1+\left\|A_{1}\right\|\right)\left\|x_{k-1}\right\|_{\infty}+q\right\} \\
& \leqq\left\|A_{1}\right\|\left\{\left(1+\left\|A_{1}\right\|\right)^{k}\left\|\varphi_{0}\right\|_{\infty}+q \sum_{j=0}^{k}\left(1+\left\|A_{1}\right\|\right)^{j}\right\} .
\end{aligned}
$$

All trajectories reaching $\varphi_{1}$ are regular, if

$$
\left\|\dot{\varphi}_{1}\right\|_{\infty}+\left\|A_{1}\right\|\left\{\left(1+\left\|A_{1}\right\|\right)^{k}\left\|\varphi_{0}\right\|_{\infty}+q \sum_{j=0}^{k}\left(1+\left\|A_{1}\right\|\right)^{j}\right\}<q .
$$

If, e.g., $\varphi_{0}=\varphi_{1}=0$ and

$$
\left\|A_{1}\right\| \sum_{j=0}^{k}\left(1+\left\|A_{1}\right\|\right)^{j}<1
$$

condition (4.2) is satisfied for all $q>0$.

This simple example illustrates that the regularity requirement for all trajectories reaching a given final function is very restrictive: Observe that (4.3) implies $\left\|A_{1}\right\|<$ $1 /(k+1)$. However, if $\Omega$ may change in time, regularity can be guaranteed if $\Omega(t)$ is big enough for $t \in T_{1}$.

The following theorem characterizes regular trajectories by a reachability property, and claims that regularity is a generic property of trajectories.

THEOREM 4.2. $x^{0} \in \mathscr{T}$ is a regular trajectory of $\Sigma_{a}$ iff in $L_{\infty}^{n}\left(T_{1}\right)$

$$
\left(\dot{x}^{0}\right)_{t_{1}} \in \operatorname{int}\left\{(\dot{x})_{t_{1}}: x \in \mathscr{T} \text { and } x(t)=x^{0}(t) \text { for } t \in\left[t_{0}-r, t_{1}-r\right]\right\} \text {. }
$$

If $\varphi_{1}$ is a regularly reachable final state of $\Lambda$ the set of regular trajectories is open and dense in $\left\{x^{0} \in \mathscr{T}: x_{t_{1}}^{0}=\varphi_{1}\right\}$.

Proof. One direction follows by Corollary 3.1. Conversely, assume that $x^{0} \in \mathscr{T}$ satisfies (4.4). By Corollary 4.1 and the construction in the proof of Theorem 4.1(ii), there is in each neighborhood of $x^{0} \in W^{n, \infty}(T)$ a regular trajectory $x^{1}$ coinciding with $x^{0}$ on $\left[t_{0}-r, t_{1}-r\right]$. By (4.4), $x^{1}$ may be chosen such that $x^{2}:=x^{0}-\left(x^{1}-x^{0}\right) \in \mathscr{T}$. Then by Lemma 4.1

$$
x^{0}=\frac{x^{1}}{2}+\frac{x^{2}}{2}
$$

is regular.

The final assertion of the theorem follows again by the construction in the proof of Theorem 4.1(ii) and linearity.

Remark 4.7. The results above illustrate that regularity occurs also for higher dimensional systems with scalar control. In Example 4.1, take the vertices $\omega_{0}, \omega_{1}, \cdots, \omega_{n}$ as images of the points $i / n$, and consider $\tilde{\Omega}:=\{0,1 / n, \cdots, 1\} \subset[0,1]$ as new control set with control actions $\omega_{i}$. More generally, condition (4.1) is, e.g., 
satisfied for the scalar control

$$
b(\omega)=\left(\begin{array}{c}
\omega \\
\omega^{2} \\
\vdots \\
\omega^{n}
\end{array}\right), \quad \text { where } \Omega(t)=\Omega_{0}:=[0,1] \quad(t \in T) .
$$

Then int co $b\left(\Omega_{0}\right) \neq \varnothing$, and hence it follows for the linear system $\Lambda$ that int $\mathscr{R} \neq \varnothing$; each element of int $\mathscr{R}$ is regularly reachable and the regular trajectories reaching $\varphi_{1}$ form an open, dense subset of the set of all trajectories reaching $\varphi_{1}$.

Remark 4.8. Let $B \subset C^{n}\left[t_{0}-r, t_{1}\right]$ and assume that there is a neighborhood $V$ of $0 \in \mathbb{R}^{n}$ such that for all $x^{0} \in B$

$$
V \subset-\dot{\varphi}_{1}\left(t-t_{1}\right)+\operatorname{co} f\left(x_{t}^{0}, \Omega, t\right) \quad\left(t \in T_{1}\right)
$$

(compare Definition 3.1). Then it appears natural to apply a fixed point theorem in order to prove local reachability of $\varphi_{1}$. In fact, if $B$ is defined by certain growth conditions on $f$, and a finite dimensional condition for the reachability of $\varphi_{1}(-r)$ at time $t_{1}-r$ is added, one can prove such a result using Kakutani's fixed point theorem (Colonius [8, Thms. 3.1, 3.3]). For Example 4.1, this yields that $\varphi_{1}=0$ is locally reachable [8, Beispiel 4.1]. In the context of control theory, this classical argument was used, e.g., by Tarnove [25]. Angell [1] and Chukwu [6], [7] applied it to hereditary differential systems in order to achieve reachability of a fixed final state.

Acknowledgment. I thank Diederich Hinrichsen for many fruitful discussions. This paper originated from my doctoral thesis which was guided by him.

\section{REFERENCES}

[1] T. S. ANGELL, Existence theorems for a class of optimal control problems with delay, Doctoral thesis, University of Michigan, Ann Arbor, 1969.

[2] H. T. BANKS, M. Q. JACOBS, AND C. E. LANGENhop, Function space controllability for linear functional differential equations, Differential Games and Control Theory, E. O. Roxin, P. Liu and R. L. Sternberg, eds., Marcel Dekker, New York-Basel, 1974, pp. 81-98.

[3] - Characterization of the controlled states in $W_{2}^{(1)}$ of linear hereditary systems, SIAM J. Control, 13 (1975), pp. 611-649.

[4] G. R. BATES, Hereditary optimal control problems, Ph.D. thesis, Purdue University, W. Lafayette, IN, 1977.

[5] L. D. Berkovitz, A penalty function proof of the maximum principle, Appl. Math. Optim., 2 (1975/6), pp. 291-303.

[6] E. N. CHUKWU, Functional inclusion and controllability of nonlinear neutral functional differential systems, J. Opt. Theory Appl., 29 (1979), pp. 291-300.

[7] — Controllability of delay systems with restrained controls, J. Opt. Theory Appl., 29 (1979), pp. 301-320.

[8] F. Colonius, Hereditäre differenzierbare Systems mit Funktionenraum-Endbedingung und punktweisen Steuerbeschränkungen: Notwendige Optimalitätsbedingungen und Erreichbarkeit, Dissertation, Universität Bremen, Bremen, 1979.

[9] - Regularization of Lagrange multipliers for time delay systems with fixed final state, in Optimization and Optimal Control, A. Auslender, W. Oettli and J. Stoer, eds., Springer-Verlag, BerlinHeidelberg-New York, 1981, pp. 163-177.

[10] - The maximum principle for relaxed hereditary differential systems with function space end condition, this Journal, this issue, pp. 695-712.

[11] F. COLONIUS AND D. HINRICHSEN, Optimal control of functional differential systems, this Journal, 16 (1978), pp. 861-879.

[12] M. C. Delfour AND S. K. MitTer, Controllability, observability, and optimal feedback control of affine hereditary differential systems, SIAM J. Control, 10 (1972), pp. 298-328. 
[13] N. Dunford AND J. T. SchwARTZ, Linear Operators, Part I: General Theory, Wiley-Interscience, New York, 1967.

[14] J. HALE, Theory of Functional Differential Equations, second ed., Springer-Verlag, Berlin-HeidelbergNew York, 1977.

[15] M. Q. JacobS AND T. J. KAO, An optimum settling problem for time lag systems, J. Math. Anal. Appl., 40 (1972), pp. 687-707.

[16] S. KuRCYUSz, On the existence and nonexistence of Lagrange multipliers in Banach spaces, J. Opt. Theory Appl., 20 (1976), pp. 81-110.

[17] S. KuRCYUSZ AND A. W. OLBROT, On the closure in $W^{1, a}$ of the attainable subspace of linear time lag systems, J. Differential Equations, 24 (1977), pp. 29-50.

[18] E. B. LeE AND L. MARkus, Foundations of Optimal Control Theory, John Wiley, New York, 1967.

[19] A. MANitius, Necessary and sufficient conditions of approximate controllability for general linear retarded systems, this Journal, 19 (1981), pp. 516-532.

[20] A. MANITIUS AND R. TRIGGIANI, Function space controllability of linear retarded systems, a derivation from abstract operator conditions, this Journal, 16 (1978), pp. 599-643.

[21] I. P. NATAnson, Theorie der Funktionen einer reellen Veränderlichen, Akademie Verlag, Berlin, 1961.

[22] M. N. Oguztöreli, Time-Lag Control Systems, Academic Press, New York, 1966.

[23] A. W. Olbrot, Control of retarded systems with function space constraints, part 2: approximate controllability, Control and Cybernetics, 6 (1977), pp. 17-71.

[24] A. B. SCHWARZKOPF, Relaxed control problems with state equality constraints, SIAM J. Control, 13 (1975), pp. ó77-694.

[25] I. TARnOVE, A controllability problem for nonlinear systems, in Mathematical Theory of Control, A. V. Balakrishnan and L. W. Neustadt, eds., Academic Press, New York, 1967.

[26] J. WARGA, Optimal controls with pseudo-delays, SIAM J. Control, 12 (1974), pp. 286-299.

[27] — Optimal Control of Differential and Functional Equations, Academic Press, New York, 1972. 
Reproduced with permission of the copyright owner. Further reproduction prohibited without permission. 